\title{
Releasing severe adhesions around the eminence and the synovial portion of the TMJ: A clinical study of combined treatment using hydraulic lavage, arthroscopic surgery and rehabilitative therapy
}

\author{
Minoru Hori, Masahiro Okaue, Daisuke Harada, Masaru Ono, Toshiyuki Goto, \\ Yusuke Tada, Daisuke Kamogawa, Mitsuhiko Matsumoto and Hiroshi Tanaka \\ Department of Oral and Maxillofacial Surgery, Nihon University School of Dentistry, Tokyo 101-8310
}

(Received 15 February and accepted 6 May 1999)

\begin{abstract}
This paper evaluates the effects of treatment with a pumping technique and arthroscopic lysis and lavage, followed by rehabilitative training, on condylar head mobility of the temporomandibular joint (TMJ). We studied 32 TMJs in 19 patients suffering from chronic closed lock with severe adhesion. The results were compared between cases with adhesions concentrated in two areas: mostly in the posterior and/or the anterior synovial portion of the upper TMJ compartment (11 joints) and mostly around the eminence ( 21 joints). The results showed a statistically significant improvement in condylar head movement for both groups between the initial and final stages of treatment. However, the results also suggested that patients with adhesion concentrated around the eminence are less likely to recover condylar head mobility to the same extent as those in the other group. (J. Oral Sci. 41, 61-66, 1999)
\end{abstract}

Key words: intracapsular fibrosis; arthroscopic surgery; condylar head movement; mouth opening.

\section{Introduction}

Internal derangement of the temporomandibular joint (TMJ) consists of displacement or malpositioning of the disk in relation to the condylar head and the articular eminence (14). It can be a major cause of closed lock of the TMJ, which is either acute or chronic. In acute cases, there is pain and severe limitation of mouth opening, caused by malpositioning of the disk (5). In some chronic cases, derangement of the TMJ may eventually lead to intracapsular microbleeding and effusion, which, in turn, can result in disk surface friction and fibrillation, with subsequent adhesion in the superior compartment of the TMJ (6). This paper focuses on chronic cases of closed lock with severe adhesion.

In chronic cases of TMJ closed lock, dysfunction of the mandible is sometimes made more severe by secondary

Correspondence to Dr. Minoru Hori, Department of Oral and Maxillofacial Surgery, Nihon University School of Dentistry, 1-8-13 Kanda-Surugadai, Chiyoda-ku, Tokyo 101-8310, Japan. pathology, such as intra-articular adhesion (5). In the past, open surgery to reposition the disk without reduction was recommended (7). However, an arthroscopic approach has recently become the method preferred by many surgeons for both diagnostic and therapeutic procedures in chronic closed lock of the TMJ, as it can release adhesions with minimal damage to joint structures (8). A number of different techniques of arthroscopic surgery have been reported (3, 9-17); they have variable success rates, depending on both the technique and the individual patients' circumstances.

This paper evaluates the results of a combination of a pumping technique and arthroscopic surgical lysis and lavage, followed by rehabilitative training. A total of 32 joints were studied in 19 patients, all of whom were suffering from chronic closed lock of the TMJ involving severely restricted translation of the condylar head caused by severe intra-articular adhesion, as indicated by arthrographic diagnosis. The success rate was determined through follow-up radiographic studies of the effect of treatment on condylar movement and mouth-opening. In all cases, the pumping lavage technique was first applied to try to release fine adhesions before carrying out arthroscopic surgery using electrocautery.

\section{Materials and Methods}

\section{Patient Selection and Treatment Procedure}

From 1991 to 1997, a total of 85 patients displaying symptoms of disk dysfunction underwent cine fluoroarthrography of the TMJ using lateral oblique transcranial and transorbital projections, in which Omnipaque water-soluble nonionic iodine contrast medium (containing iodine $350 \mathrm{mg} / \mathrm{ml}$ ) was injected into the upper and lower compartments of the TMJ, in order to diagnose the causes of the dysfunction. Those patients diagnosed as having the greatest limitation of disk mobility, as well as severe adhesion in the TMJ, received hydraulic lavage with a contrast medium to expand the compartment and improve the mobility of the condylar head and degree of mouth opening. For some patients, this technique provided satisfactory improvement in the degree of mouth opening and smooth translation of the condylar head past the eminence, as confirmed by standardized bilateral oblique transcranial radiography. 
However, about half the patients continued to experience restriction of the movement of the condylar head to the intramandibular fossa or to beneath the eminence, as confirmed by a second standardized bilateral oblique transcranial radiograph; it was recommended that these patients undergo arthroscopic surgery to release adhesions. Of the patients who were advised to undergo this treatment, 19 agreed to do so: 18 women and 1 man, all Japanese, aged between 21 and 69 years, with a mean age of 46.9 years. This paper describes the changes in condylar head movement and mouth opening at each stage of treatment of these 19 patients with severely restricted intra-articular movement of the condyle. Arthrographic diagnosis indicated the presence of severe adhesions in the upper compartment of 32 TMJs in this group of patients, and these 32 TMJs formed the basis of this study. The severe adhesions were located in the anterior recess, with concomitant disk displacement without reduction. In addition, there was a marked fibrous and inflammatory response of adhesions running from both the synovial surface and the articular surface above, and to the synovial and disk surfaces below.

Using a Stryker mini-arthroscopic system, and with the patients under general anesthesia administered via nasotracheal intubation, a 2.3- $\mathrm{mm}$ cannula was inserted into the upper compartment of the TMJ to examine the joint cavity with a zero-degree endoscope. The adhesions were released with a
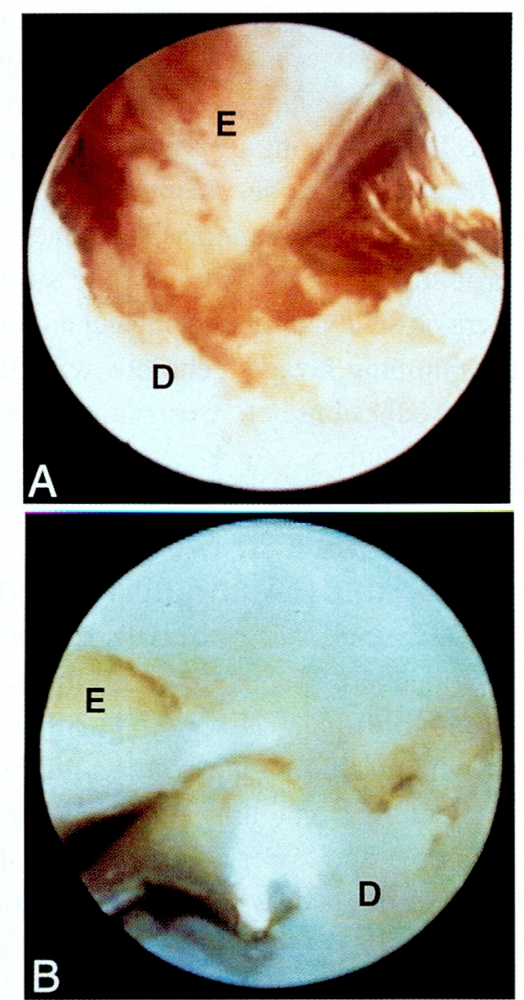

Fig. 1 Close-up view of arthroscopic surgery.

$$
\text { D: disk E: eminence }
$$

A: Blunt obturator performing lysis and release of a disk osseous band, allowing expansion of the superior compartment.

B: Use of electrocautery to release a severe disk osseousband adhesion between the eminence and the disk. blunt trocar and an electric cauterizer inserted through a second cannula, using McCain's technique to determine the second point and the angulation of entry (Fig. 1). This placed the second puncture in the most anterolateral aspect possible, given the pathology of each joint. In the medial section of the upper compartment it was possible to use visual techniques for releasing the adhesions. Weak adhesions, such as interosseous fibrosis, were released with a blunt obturator alone, followed by lysis (Fig. 2), while severe adhesions were released with an electric cauterizer. As part of this procedure, the synovial drape was cut, exposing the underlying lateral pterygoid muscle fibers, of which the superficial fibers were cut to provide release. In the lateral section of the compartment only blind techniques could be used to release the adhesions. Therefore, both weak and severe adhesions were treated with a combination of the lateral eminence release technique and the capsular stretch technique.

For all 19 patients, rehabilitative training to improve mouthopening was begun 2-3 weeks after surgery, and continued with decreasing frequency for the following 3-12 months, according to the needs of each case. The treatment goal was to achieve satisfactory functional mobility of the TMJ, defined as adequate condylar movement and the ability to open the mouth at least $40 \mathrm{~mm}$ without discomfort. The rehabilitative training was performed using a mouth gag and/or Therabite jaw motion
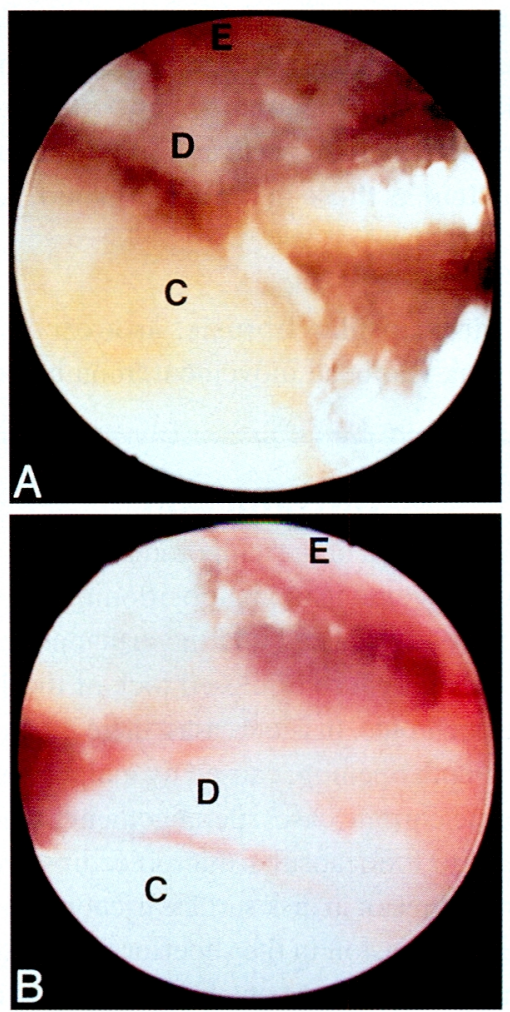

Fig. 2 Treatment of interosseous fibrosis.
D: disk
E: eminence
C: condylar head

A: Exposed condylar head showing large perforation with fibrillation in the retrodiskal area allowing access to the inferior compartment.

B: Blunt obturator performing lysis of a dhesion; tip is inserted to allow access to superior compartment. 
rehabilitation equipment, together with manipulation to achieve forward and contralateral guided movement of the mandible.

\section{Evaluation}

For radiographic evaluation of condylar head movement,standardized bilateral oblique transcranial radiographs were taken of each TMJ before treatment, after application of the pumping technique, 2-3 weeks after arthroscopic surgery, and after 3-12 months of excursive training in mandibular movement. Tracings of the radiographs with reference to the processus retroarticularis and the tuberculum articulare determined the location of the mandibular fossa. After further tracings of the top of the condylar head were made, measurements were taken in order to assess changes in translated movement of the condylar head. A line ' $a-b$ ' was drawn to show the total degree of condylar movement (Fig. 3). The degree of mouth-opening at each stage was measured with calipers.

In the 32 joints examined in this study, several types of adhesion, classified according to Kaminishi's definitions (19), were found in all cases (Figs 2 and 4). However, the joints could be divided into two groups, according to the main location of the adhesions. In Group A (11 joints), adhesions mostly occurred in the posterior and/or the anterior synovial portions of the upper TMJ compartment. In Group B (21 joints), the adhesions were concentrated around the eminence, occurring in one or more of the following positions: between the disk and the eminence, between the retrodiskal tissue and the posterior slope of the eminence, and between the anterior recess and the anterior slope of the eminence. In 2 patients both joints were classified in Group A; in 7 patients 1 joint was classified in Group B; in 4 patients both joints were classified in Group B, and in the remaining 6 patients 1 joint was classified in Group A and the other in Group B.

Simple fibrous bands were found in the usual locations: from the eminence to the disk or the retrodiskal tissue. Similarly, fibrosynovial bands were found in the anterior and the posterior recess, or between the fossa or the eminence and the retrodiskal tissue. Early fibrosis, or filmy adhesion, occurred only between the eminence and the disk and/or the retrodiskal tissue, and lateral capsular fibrosis was also present. All of these types of adhesion could be released relatively easily with a blunt trocar

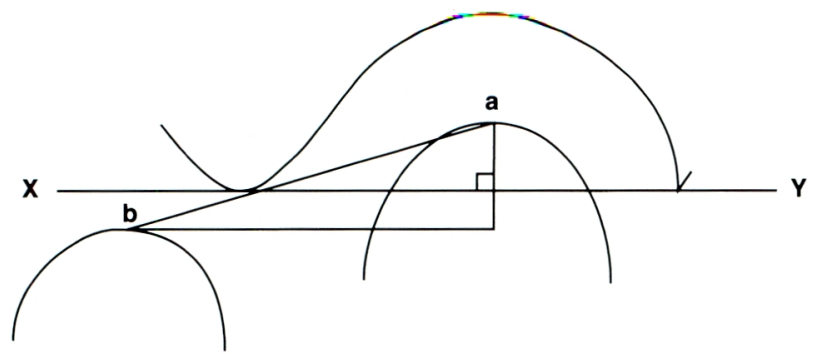

Fig. 3 Schematic drawing of TMJ showing horizontal reference line X-Y through the base of the articular eminence to the squamotympanic fissure. Points a and $\mathrm{b}$ represent the position of the superior condylar point at the closed jaw position and maximal mouth opening. The distance between points $\mathrm{a}$ and $\mathrm{b}$ was measured to evaluate condylar mobility.

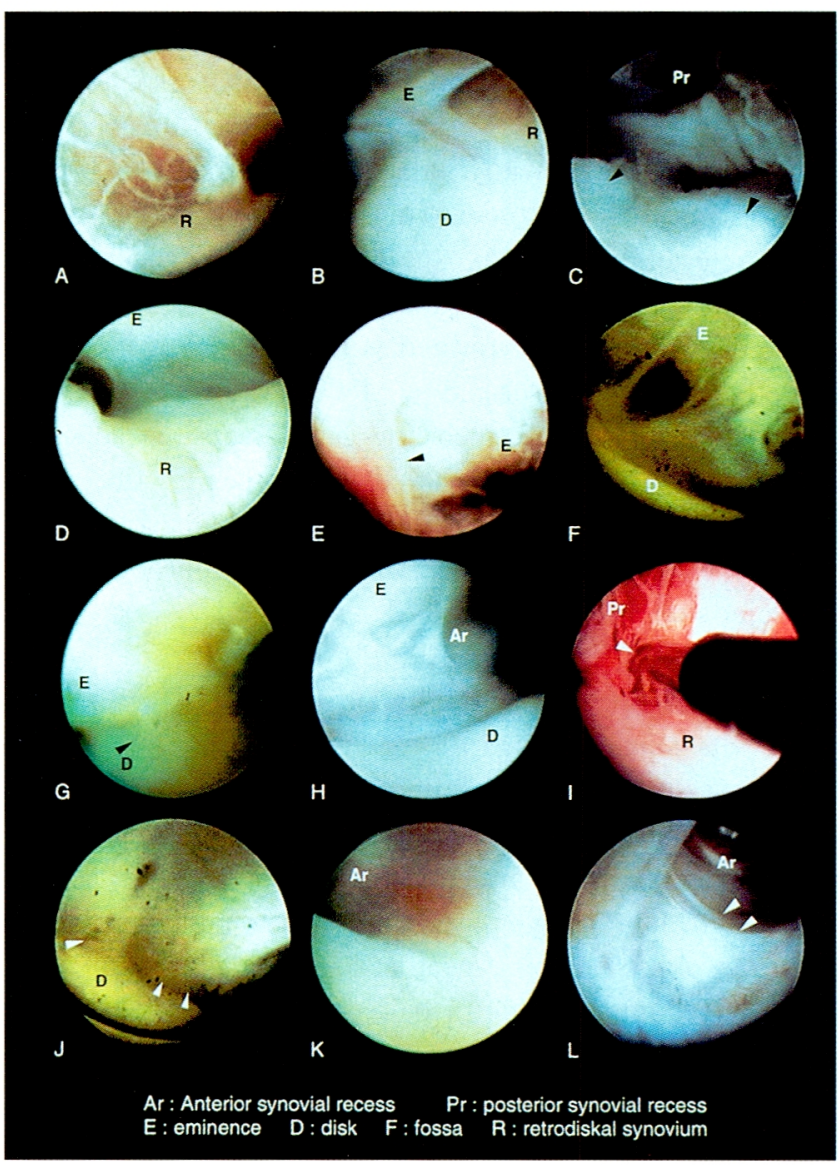

Fig. 4 Arthroscopic view (A-L).

A: Simple fibrous from synovium of the posterior pouch to terodiskal tissue and dense fibrous band.

B: Fibrosynovial band, with visible capillaries, from eminence to border between disk and retrodiskal tissue, lack of dense fibrous bands indicating possibly immature adhesion.

C: Fibrosynovial bands from synovium of the posterior pouch to posterior medial aspect of disk and posterior slope of eminence. Arrow shows extensive redundancy.

D: Early fibrosis from posterior slope of eminence to retrodiskal synovium. Filmy adhesion has been broken with probe.

E: Arrow shows lateral capsular fibrosis

F: Diskal osseous band. Well-defined adhesion between eminence and disk.

G: Arrow shows disk osseous band from eminence to disk. Degenerative cartilage and bleeding can be seen on the surface of the eminence.

$\mathrm{H}$ : Partial fibrous and fibrosynovial pseudowall from anterior slope of eminence to anterior synovium, completely concealing anterior recess.

I: Fibrosynovial band and partial fibrosynivial pseudowall in compartment. A possibly immature adhesive band can be seen between the synovium of posterior pouch and the retrodiskal tissue, with marked crythema.

$\mathrm{J}$ : Intermittent fibrous and fibrosynovial pseudowall from medical wall of fossa to synovium of anterior pouch and disk.

$\mathrm{K}$ : Total fibrosynovial pseudowall in anterior recess, with mild to moderate rythema.

L: Total fibrosynovial pseudowall in anterior synovial pouch, completely conccaling anterior synovium.lack of dense fibrous bands indicating possibly immature adhesion. 
and/or an electric cauterizer.

Pseudowalls with thick adhesive bands, disk osseous bands and interosseous fibrosis were more difficult to release, but an electric cauterizer with a monopolar electrocautery tip was able to achieve this. In order to release these types of adhesion in the anterior recess it was especially important accurately to select the position of the second puncture, so that a lysis maneuver could be performed with the electrocautery tip directly visible, as described by McCain (20).

Moses (14) has described how lateral capsular adhesion cannot always be directly observed. In the cases described here, a blind technique had to be used to release adhesions along the lateral aspect of the capsule; this was done through lateral capsular stretching using a blunt obturator.

\section{Results}

Results were compiled for the effects of lavage pumping treatment, surgery and rehabilitative training on the condylar head movement of joints in Group A and Group B (Fig. 5). Between the initial and the final stages, there was an improvement in condylar head mobility for both groups, with Group A improving by a mean of $5.9 \mathrm{~mm}$ and Group B by a mean of $3.6 \mathrm{~mm}$. Using the paired t-test, the treatment was seen to have more overall effectiveness in Group A. For both

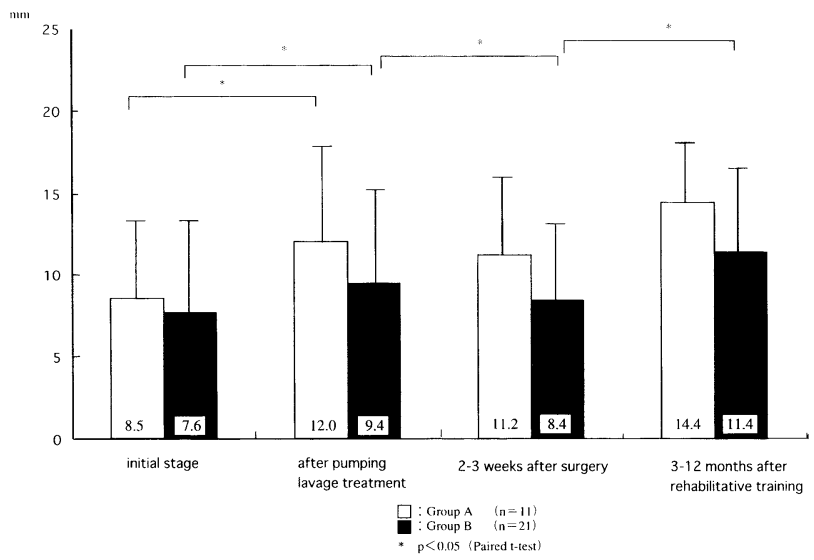

Fig. 5 Changes in condylar head movement, as revealed by rediography.

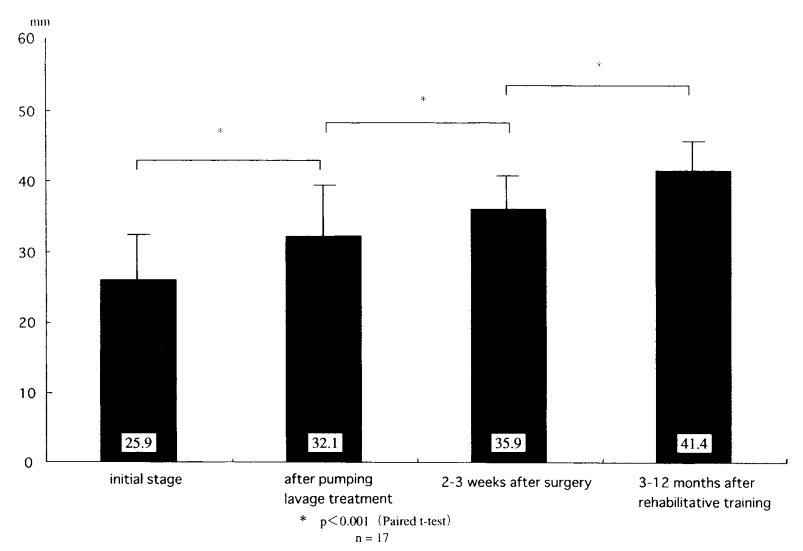

Fig. 6 Changes in maximum active opening of the mouth for 17 patients with Group B type adhesions. groups, there was an improvement in condylar head mobility in the period between first presentation and immediately after the treatment by hydraulic lavage. In both groups there was a slight increase in condylar head mobility over the period between the application of hydraulic lavage and 2-3 weeks following arthrographic surgery. In both groups mobility had improved again by 3-12 months after surgery, following rehabilitative training.

However, when we examined the effects of each part of the treatment using the paired t-test, although there was a statistically significant change between each stage of treatment for Group B, for group A only the change between the first 2 stages was significant.

The degree of improvement in mouth-opening was also examined. The number of patients whose joints were only Group-A type (2) was too small to provide statistically significant results. Therefore, measurements were taken of the 17 patients who had at least 1 joint in Group B (Fig. 6). Mouth-opening increased from a mean of $25.9 \mathrm{~mm}$ at the initial stage to a mean of $32.1 \mathrm{~mm}$ after application of the pumping technique. It continued to improve to a mean of 35.9 $\mathrm{mm}$ following surgery, and finally reached a mean of $41.4 \mathrm{~mm}$ in the 3-12 months afterward. Paired t-tests showed statistically significant changes between each of these stages.

\section{Discussion}

In Japan, approximately 10-20\% of patients with chronic closed lock of the TMJ receive surgical arthroscopic treatment (21). Worldwide, the technique has often been used for arthroscopic antero-lateral capsular release, following the initial report by Sanders in 1986 (3). Most follow-up studies of the results of the procedure report a success rate of over $80 \%(3,8,9)$.

The goal of arthroscopy in cases of synovial pathology is to reduce or eliminate the inflammatory response; this is achieved by combining arthroscopic lysis and lavage with synovectomy. However, complex cases of adhesion require more than simple capsule release.

On the basis of diagnostic arthroscopy, Kaminishi (19) has classified adhesions in various locations within the TMJ upper compartment into 8 basic types:

1. Simple fibrous bands

2. Fibrosynovial bands

3. Early fibrosis

4. Capsular fibrosis

5. Disk osseous bands

6. Pseudowalls (three types)

7. Interosseous fibrosis

8. Fibro-osseous or osseous adhesions.

The cases reported in this paper presented as mixtures of 7 of these types of adhesion, excluding fibro-osseous or osseous adhesions. Other authors have suggested that fibro-osseous or osseous adhesions can be treated successfully with arthroscopic laser debridement (22).

Sanders's technique of electrocautery can be used to release adhesions between the posterior slope of the eminence and the disk, and to release synovial pseudowalls in the anterior or 
posterior recess. Severe adhesions covering a comparatively large area in the lateral section of the capsule may require the use of a blunt trocar with a blind technique, such as Moses's technique of lateral capsular release. Various other techniques, such as the use of a YAG laser for releasing adhesions, or suturing to reposition the disk, may also be used in certain cases.

The goal of all these techniques is to increase disk mobility. However, although each technique may have a high success rate when used individually, the results can sometimes be unpredictable when a combination of techniques is used in complex cases of severe adhesion. The study described here aimed to examine the results of a combination of a pumping technique, arthroscopic surgery and rehabilitative training on increasing mouth-opening and on improving disk mobility and condylar head movement in patients with adhesions focused at two different locations in the TMJ.

The results of the cases described here show a statistically significant improvement in condylar head movement in both Group A and Group B in the period between first presentation and after pumping treatment. In Group B, there was a statistically significant improvement over the period between application of the pumping technique and 2-3 weeks after surgery, as well as during the period of rehabilitative training. Group A did not show significant changes between each stage of treatment, but there was a significant improvement in condylar head movement between the initial and final stages.

These results suggest that a combination of the pumping technique, arthrographic surgery and rehabilitative training is useful in improving condylar head mobility in cases of adhesions occurring in the anterior recess and concentrated around the eminence, as in Group B, as well as for joints such as those in Group A, with adhesions mostly in the anterior or posterior synovial portion of the upper compartment. However, it also seems that joints with adhesions of the Group-B type are unlikely to recover mobility to the same extent as those with adhesions of the Group-A type.

The results also suggest that mouth-opening is likely to be improved by a combination of techniques in the types and locations of adhesion found in Group B.

\section{References}

1. Farrar, W.B. and McCarty, W.L. Jr. (1982) A clinical outline of temporomandibular joint diagnosis and treatment. 7th ed., Walker Printing, Montgomery, 5388

2. Pertes, R.A. and Attanasio, R. (1991) Internal derangements. In Temporomandibular disorders. Kaplan, A.S. and Assael, L.A. eds., W.B. Saunders Company, Philadelphia, 142-164

3. Sanders, B. (1986) Arthroscopic surgery of the temporomandibular joint: treatment of internal derangement with persistent closed lock. Oral Surg. Oral Med. Oral Pathol. 62, 361-372

4. Ohnishi, M. (1980) Clinical application of arthroscopy in temporomandibular joint diseases. Bull. Tokyo Med. Dent. Univ. 27, 141-150
5. Kaminishi, R.M. and Davis, C.L. (1989) Temporomandibular joint arthroscopic observations of superior space adhesions. Oral Maxillofac. Surg. Clin. North Am. 1, 103-109

6. Benson, B.J. and Keith, D.A. (1985) Patient response to surgical and nonsurgical treatment for internal derangement of the temporomandibular joint. J. Oral Maxillofac. Surg. 43, 770-777

7. Dolwick, M.F. and Sanders, B. (1985) TMJ internal derangement and arthrosis: surgical atlas. The C. V. Mosby Company, St. Louis, 197-215

8. Nitzan, D.W., Dolwick, M.F. and Heft, M.W. (1990) Arthroscopic lavage and lysis of the temporomandibular joint: a change in perspective. J. Oral Maxillofac. Surg. 48, 798-801

9. Moses, J.J., Sartoris, D., Glass, R., Tanaka, T. and Poker, I. (1989) The effect of arthroscopic surgical lysis and lavage of the superior joint space on TMJ disc position and mobility. J. Oral Maxillofac. Surg. 47, 674-678

10. White, R.D. (1989) Retrospective analysis of 100 consecutive surgical arthroscopies of the temporomandibular joint. J. Oral Maxillofac. Surg. 47, 1014-1021

11. Murakami, K., Segami, N., Moriya, Y. and Iizuka, T. (1992) Correlation between pain and dysfunction and intra-articular adhesions in patients with internal derangement of the temporomandibular joint. J. Oral Maxillofac. Surg. 50, 705-708

12. McCain, J.P., Podrasky, A.E. and Zabiegalki, N.A. (1992) Arthroscopic disc repositioning and suturing: a preliminary report. J. Oral Maxillofac. Surg. 50, 568579

13. Indresano, A.T. (1989) Arthroscopic surgery of the temporomandibular joint: report of 64 patients with long-term follow-up. J. Oral Maxillofac. Surg. 47, 439441

14. Moses, J.J. (1989) Lateral impingement syndrome and endaural surgical technique. Oral Maxillofac. Surg. Clin. North Am. 1, 165-183

15. Ohnishi, M. (1989) Arthroscopic surgery for hypermobility and recurrent mandibular dislocation. Oral Maxillofac. Surg. Clin. North Am. 1, 153-164

16. Kondoh, T. (1993) Arthroscopic traction suturing. Treatment of internal derangement by arthroscopic repositioning and suturing of the disk. In Advances in diagnostic and surgical arthroscopy of the temporomandibular joint. Clark, G.T., Sanders, B. and Bertolami, C.N. eds., W.B. Saunders Company, Philadelphia, 117-127

17. Zeitler, D.L. and Porter, B.T. (1993) A retrospective study comparing arthroscopic surgery with arthrotomy and disk repositioning. In Advances in diagnostic and surgical arthroscopy of the temporomandibular joint. Clark, G.T., Sanders, B. and Bertolami, C.N. eds., W.B. Saunders Company, Philadelphia, 47-60 
18. Okaue, M., Hori, M., Satoh, H., Harada, D., Ono, M., Tada, Y., Goto, T., Kamogawa, D., Matsumoto, M. and Tanaka, H. (1998) A study of the effects of hydraulic lavage and arthroscopic treatment on condylar movement and mouth opening in cases of closed lock of the temporomandibular joint. Nihon Univ. Dent. J. $72,832-840$ (in Japanese)

19. Kaminishi, R.M. (1993) Intracapsular fibrosis related to pain and dysfunction. In Advances in diagnostic and surgical arthroscopy of the temporomandibular joint. Clark G.T., Sanders B. and Bertolami C.N. eds., W.B. Saunders Company, Philadelphia, 5-14
20. McCain, J.P. and Menis, M.A. (1996) Puncture techniques and arthroscopic anatomy. In Principles and Practice of the temporomandibular joint arthroscopy. McCain J.P. ed., Mosby,St.Louis, 128-165

21. Segami, N., Murakami, K., Hosaka, H. and Iizuka, T. (1993) Arthroscopic antero-lateral capsular release for internal derangement of the temporomandibular joint. Kansetsukyou 18, 105-110 (in Japanese)

22. Moses, J.J., Lee, J. and Arredondo, A. (1998) Arthroscopic laser debridement of temporomandibular joint fibrous and bony ankylosis: case report. J. Oral Maxillofac. Surg. 56, 1104-1106 